\title{
Modelación del cambio de uso del suelo en Comalcalco, Tabasco, México
}

\author{
Modelling of the change of land use in Comalcalco, Tabasco, México
}

Rodimiro Ramos Reyes *

Miguel Ángel Palomeque de la Cruz**

Fecha de recepción: 03 de julio de 2017

Fecha de aceptación: 25 de octubre de 2017

\section{Resumen}

El cambio de uso del suelo en Comalcalco, Tabasco, México, se modeló teniendo como base el efecto provocado por el crecimiento de las actividades antropogénicas sobre las coberturas naturales. Se hizo un análisis espacial mediante el Modelador de Cambios en el Terreno (MCT) y se calcularon tasas de cambio. Durante los años 2000-2010 se perdieron 8.391 ha de vegetación hidrófita y 1.048 ha de cuerpos de agua, ante el crecimiento acelerado de 8.391 ha de uso agrícola y 810 ha de la zona urbana. Se destacó la pérdida de 3.050 ha de pastizal y el aumento de 1.415 ha de manglar. Las Cadenas de Markov y los Autómatas Celulares proyectaron un escenario (2030) en donde se pierden 544 ha de vegetación hidrófita, 1.048 ha de cuerpos de agua y 3.050 de pastizal, ante los aumentos de 2.425 ha de uso agrícola, 1.415 ha del manglar y 1.110 de la zona urbana. El análisis espacial y la proyección resaltan la importancia de proteger la vegetación hidrófita, los cuerpos de agua y las crecientes superficies de manglar mediante una planificación ambiental que establezca formalmente zonificaciones definidas como: Área Natural Protegida, Conservación, Prioritaria de Conservación y Aprovechamiento, como lo señala el Programa de Ordenamiento Ecológico.

Palabras clave: Autómatas Celulares, Cadenas de Markov Comalcalco, Modelador de Cambios en el Terreno, planificación ambiental, Tabasco.

\begin{abstract}
The land use change in Comalcalco, Tabasco, México, was modeled on the effect of the growth of anthropogenic activities on natural coverages. A spatial analysis was done using the Land Change Modeler (LCM) and exchange rates were calculated. During 2000-2010, 8,391 ha of hydrophilic vegetation and 1,048 ha of water bodies were lost, due to the accelerated growth of 8,391 ha of agricultural use and 810 ha of urban areas. He noted the loss of 3,050 ha of pasture and the increase of 1,415 ha of mangrove. The Markov Chains and the Cellular Automata projected a scenario (2030) where 544 ha of hydrophilic vegetation, 1,048 ha of water bodies and 3,050 of pastureland were lost, due to the increase of 2,425 ha of agricultural use, 1,415 ha of mangrove and 1.110 of the urban area. Spatial analysis and projection highlight the importance of protecting hydrophilic vegetation, water bodies and increasing mangrove areas through an environmental planning that formally establishes zoning defined as: Protected Natural Area, Conservation, Priority Conservation and Utilization, as indicates the environmental management program.
\end{abstract}

Keywords: cellular automata, Comalcalco, environmental planning, Land Change Modeler, Markov Chains, Tabasco.

\footnotetext{
* Filiación: El Colegio de la Frontera Sur, Unidad Villahermosa, Tabasco, México. Correo electrónico: rrreyes73@hotmail.com

** Filiación: Universidad Juárez Autónoma de Tabasco, División Académica de Ciencias Biológicas, Villahermosa, Tabasco, México. Correo electrónico: migueldacbiol@hotmail.com
}

Cómo citar: Ramos, R. y Palomeque, M. (2017). Modelación del cambio de uso del suelo en Comalcalco, Tabasco, México. Revista de Urbanismo, 37, 1-17. https://doi.org/10.5354/0717-5051.2017.47986 


\section{Introducción}

\section{El cambio de uso del suelo en el mundo}

El cambio de uso del suelo es una de las mayores presiones crecientes de las acciones humanas que afecta gran parte del planeta (Newbold et al., 2015). La agricultura, industria, silvicultura y el establecimiento de ciudades son las acciones más comunes que aceleran estos cambios (Akinbobola, 2015; Miller, Hauer \& Werner, 2015; Munteanu et al., 2014). El aumento de la producción agrícola para alimentar a la población mundial ha contribuido a la conversión de bosques y está generando, en amplias escalas geográficas, una fuerte presión y efectos negativos sobre la estructura y funcionamiento de los biomas, siendo los más afectados los ecosistemas tropicales (Challenger et al., 2009; Godfray et al., 2010; Klanderud et al., 2010).

En el planeta se registra un cambio de 136 a 658 millones de hectáreas desde el año 1700 al 2000, destacando la conversión a pastizal con 418 millones de ha (Goldewijk, Beusen, Drech, \& de Vos, 2011). De la misma forma, ha desaparecido alrededor del $50 \%$ de superficies ocupadas originalmente por humedales (Mitsch, Goseelink \& Anderson, 2009; Torbick, Qi, Roloff \& Stevenson, 2006). En 1990 los bosques cubrían el $31,6 \%$ del planeta (4.128 millones de ha) pero en 2015 disminuyeron a 30,6 \% (3.999 millones de ha). Esto quiere decir que en 15 años se han perdido 129 millones de hectáreas de bosques en todo el mundo (Organización de las Naciones Unidas para la Alimentación y la Agricultura, FAO, 2015). Estos cambios están generando inundaciones, erosión del suelo, degradación de la calidad de agua y pérdidas en los servicios de los ecosistemas que contribuyen a la vulnerabilidad ante el cambio climático (Amatya, Skaggs \& Trettin, 2009; Sun \& Lockaby, 2012).

\section{Deterioro de los ecosistemas en México}

En México se están deteriorando bosques, desiertos y humedales cercanos a ecosistemas urbanos; por ejemplo, en la Ciudad de México, una extensa superficie de reservas naturales ha sido transformada en zonas urbanas y más del 30\% de los humedales desaparecieron (Torres-Vera, Prol-Ledesma \& García-López, 2009; Zepeda-Gómez; Nemiga, Lot Helgueras y Madrigal Uribe, 2012). Como consecuencia de los niveles de urbanización acelerados en México, durante 1976-2007, se presentaron cambios de uso del suelo en todas las regiones, con una superficie anual deforestada de 534.707 ha, y una tasa anual de 0,08\% para bosques, y $0,41 \%$ para selvas (Rosete-Vergés et al., 2014). Informes de FAO (2015) reportan elevadas tasas de deforestación de bosques templados y tropicales de 0,3\% entre 1990 2015. Los humedales perdieron el $62,1 \%\left(69.684 \mathrm{~km}^{2}\right)$ de la cobertura nacional durante 2002-2008, y más de la mitad de los estados han perdido el $50 \%$ de sus humedales (Brena, Castillo y Wagner, 2016).

\section{Cambio de uso del suelo en Tabasco}

En el estado de Tabasco, a mediados del siglo XX sobresalieron dos etapas en relación con la forma de organización de la economía, los cuales estuvieron caracterizados por la creación de nuevos centros urbanos y el crecimiento de algunos existentes, con aceleradas tasas de deforestación, en los que destacan: la ganadería extensiva dirigida al mercado nacional que incluyó el Plan Chontalpa (1965-1976), y el Programa de Desarrollo Rural Integrado para el Trópico Húmedo, en los municipios de Cárdenas, Comalcalco, Centla, Paraíso y Macuspana; y el auge petrolero que inició en los años 70, mismo que se mantuvo hasta la década de los 80 (Arrieta, 1994; Capdepont-Ballina y Marín-Olán, 2014; Flores-Santiago, 1987; Lezama, 1987).

Ahora bien, el cambio de uso del suelo en la región de la Chontalpa, Tabasco, está relacionado con el crecimiento de la población, principalmente en las décadas de los años 60 y 70, la actividad agropecuaria, explotación forestal, explotación petrolera y el crecimiento de las zonas urbanas (Capdepont-Ballina y Marín-Olán, 2014; Lezama, 1987). Las actuales zonas urbanas anteriormente fueron ocupadas por agricultura de temporal y por pastizales que aumentaron a costa de los tulares, manglares y agroecosistemas, lo que trajo la pérdida de asociaciones vegetales como el palmar y la selva alta perennifolia, transformándose en grandes áreas ganaderas, con efectos ecológicos que impiden el flujo e intercambio de organismos, con lo que se debilita la conectividad y disminuyen las poblaciones y las cadenas bióticas (Capdepont-Ballina y Marín-Olán, 2014). En el sureste de México también se presentan grandes tasas de deforestación del manglar, y la gran superficie de costas hace imposible estimar con exactitud la tasa de pérdida en el paso del tiempo (Hirales-Cota, EspinozaAvalos, Schmook, Ruiz-Luna y Ramos-Reyes, 2010). 


\section{Modelos geomáticos del cambio de uso del suelo}

La información respecto de la dinámica de los ecosistemas, las estrategias de conservación y la restauración son esenciales para la planificación ambiental (Rawat \& Kumar, 2015). Por su parte, la investigación acerca del modelamiento espacio-temporal en territorios urbanizados, rurales o naturales, es importante para predecir escenarios ambientales y socioeconómicos, con la finalidad de evaluar las políticas de manejo territorial (Al-shalabi et al., 2013; Berberoğlu Akın, \& Clarke, 2016). Es aquí donde cobra relevancia el Modelador de Cambios en el Terreno (MCT), que está orientado al constante problema de conversión acelerada del terreno y a las necesidades analíticas de la conservación de la biodiversidad (Eastman, 2012). En el $\mathrm{MCT}$, las herramientas están organizadas en: análisis de cambios, predicción de cambios, evaluación del hábitat y su impacto sobre la biodiversidad e intervenciones de planeación (Eastman, 2012).

Por su parte, las Cadenas de Markov simulan la predicción del estado de un sistema en un tiempo determinado a partir de dos estados precedentes (Eastman, 2012). Es un procedimiento discreto en un tiempo discreto, en donde el valor en el tiempo t1 depende de los valores en los tiempos t0 y $\mathrm{t}-1$. La predicción se materializa en una serie de mapas de usos del suelo para un tiempo futuro, en donde el nivel digital de cada píxel expresa la probabilidad de pertenecer a la categoría analizada (Al-shalabi et al., 2013; Berberoğlu et al., 2016; Eastman, 2012). En este escenario, los Autómatas Celulares funcionan como un conjunto de elementos idénticos, llamado "células", cada una de las cuales se encuentra en un espacio discreto. Dichas unidades espaciales contienen una historia y una evolución de cambio en el tiempo, además de reglas como la influencia de celdas colindantes a una celda central (Al-shalabi et al., 2013; Berberoğlu et al., 2016; Clarke \& Gaydos, 1998).

\section{Objetivo del estudio y preguntas de investigación}

Es importante mencionar que, en gran parte de Tabasco, existe una carencia de datos acerca del estado actual de la dinámica espacial de las coberturas de vegetación natural y de los humedales por crecimiento urbano y agropecuario, a pesar de tratarse de información relevante para sustentar escenarios del cambio de uso del suelo en corto y mediano plazo, que permitan la planificación ambiental para restablecer servicios y beneficios ambientales (Palomeque-De la Cruz et al., 2017). Ante este panorama, el objetivo de estudio fue analizar el cambio de uso del suelo en Comalcalco, Tabasco (2000-2010), identificando las principales transiciones entre las coberturas naturales y los usos antropogénicos, empleando para ello el MCT. También se proyectaron probabilidades de cambio de uso del suelo y un escenario espacial (2030), empleando Cadenas de Markov y Autómatas Celulares, con la finalidad de generar un diagnóstico actual y prospectivo que contribuya con las actuales estrategias de planeación ambiental.

Las preguntas de investigación fueron las siguientes:

- ¿Cuáles son las principales transiciones espaciales durante 2000 y 2010, entre las coberturas naturales y los usos antropogénicos?

- ¿Cuál es la probabilidad de que las coberturas naturales y usos antropogénicos pierdan o aumenten sus áreas en el 2030?

- ¿Qué estrategias de planificación ambiental en 3 el municipio se han propuesto para conservar las coberturas naturales que aún persisten?

\section{Metodología}

\section{Área de estudio}

El municipio de Comalcalco, en el estado de Tabasco, México (Figura 1), se ubica en la región Chontalpa, entre las coordenadas $18^{\circ} 09^{\prime} \mathrm{LN}$ y $92^{\circ} 32^{\prime} \mathrm{LO}$; con superficie de $723.19 \mathrm{~km}^{2}$; clima cálido húmedo con abundantes lluvias en verano; temperatura media anual entre 24 y $28^{\circ} \mathrm{C}$; y precipitación media anual entre 1500 y $3000 \mathrm{~mm}$ (Instituto Nacional de Estadística, Geografía e Informática, INEGI, 2006). 


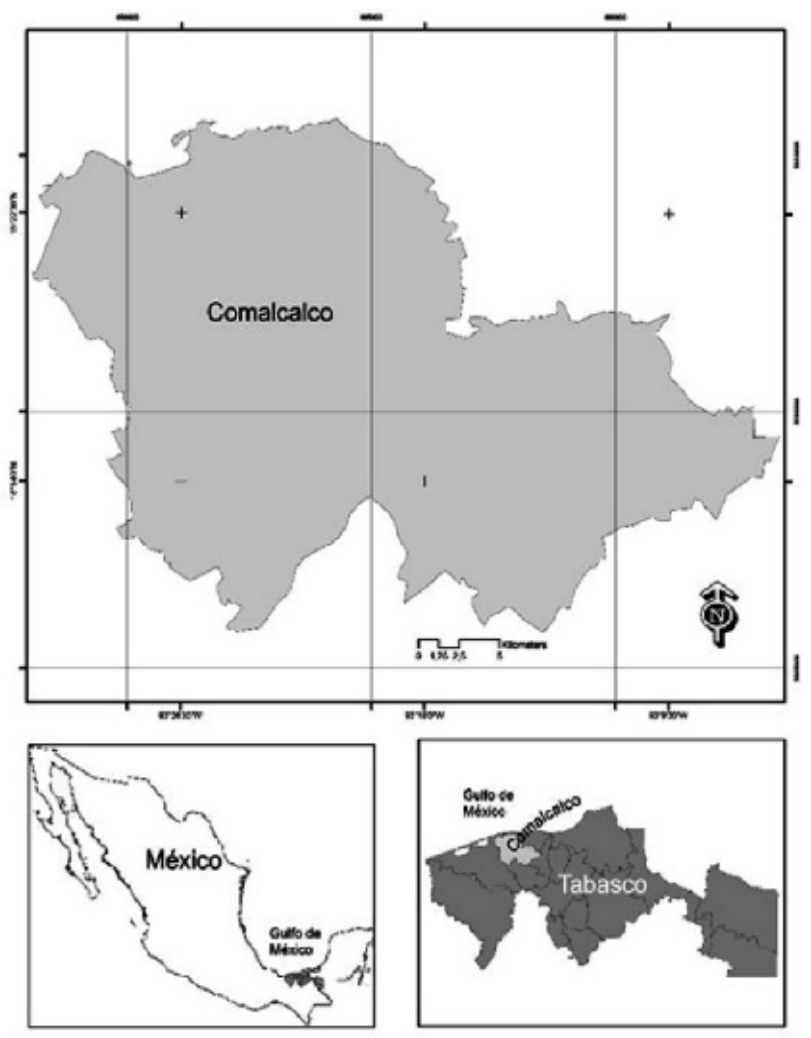

Figura 1. Municipio de Comalcalco en el Estado de Tabasco, México.

Fuente: Elaboración propia a partir de datos de (INEGI, 2006).

\section{Base de datos geográficos}

Se generaron dos mapas de uso del suelo 2000 (Figura 2) y 2010 (Figura 3), Datum WGS84-Proyección UTM zona $15 \mathrm{~N}$ en formato vectorial, elaborados mediante digitalización en pantalla, y posteriormente trasformadas en formato raster. Así mismo, se emplearon los programas Quantum GIS ${ }^{\circ}$, ArcView $3.2^{\circ} \mathrm{y}$ ArcGIS $9.2^{\circ}$. Los usos antropogénicos identificados fueron: agricultura, pastizal, y urbano; mientras que las coberturas naturales fueron: vegetación hidrófita, manglar y cuerpos de agua.

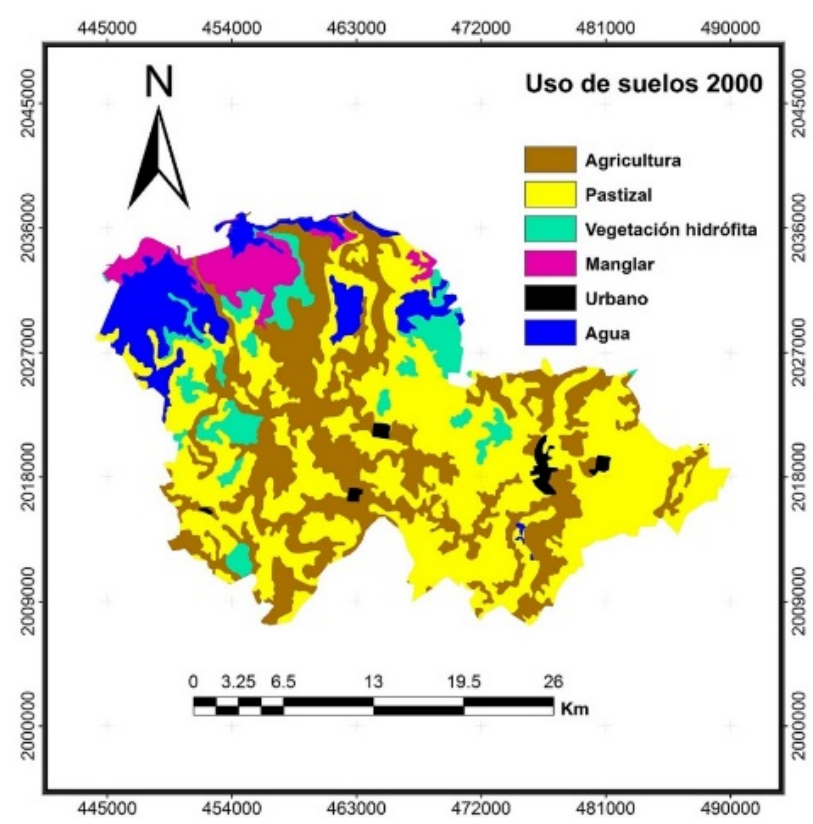

Figura 2. Mapa de uso de suelos en Comalcalco (2000).

Fuente: Elaboración propia.

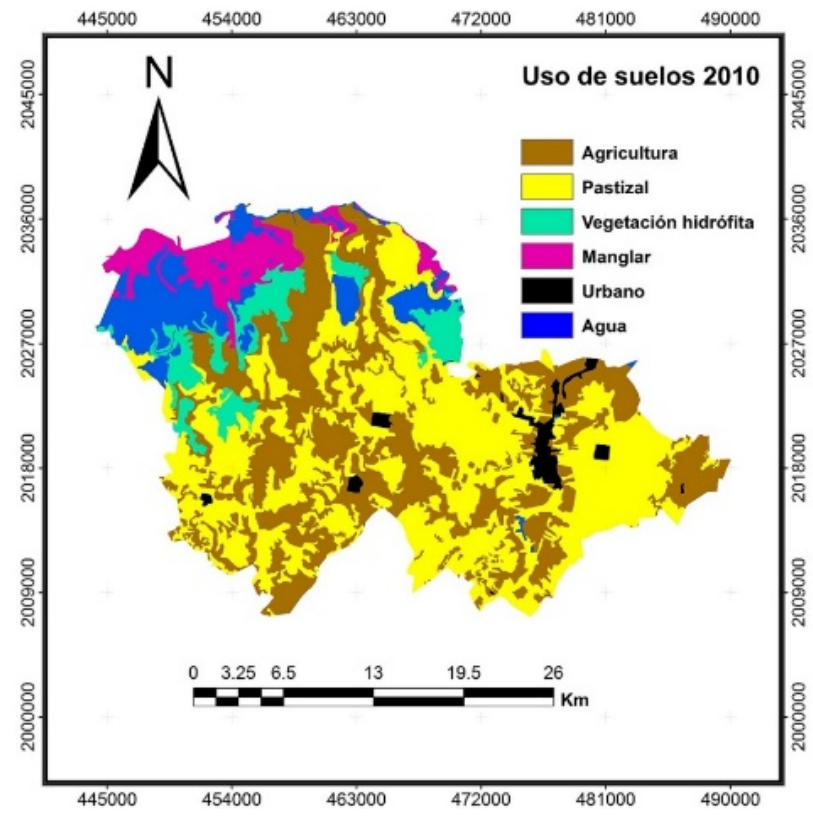

Figura 3. Mapa de uso de suelos en Comalcalco (2010).

Fuente: Elaboración propia.

\section{Modelador de Cambios en el Terreno, MCT}

El análisis espacio-temporal con los mapas del 2000 (Figura 2) y 2010 (Figura 3), se realizó con el MCT y el módulo CrossTab del software IDRISI Selva (Eastman, 2012). Los resultados incluyen el resumen de las matrices que muestran la superficie de cada categoría en 
comparación con otras, en términos de ganancias, pérdidas y contribuciones entre categorías (Eastman, 2012).

\section{Tasas de cambio}

Las tasas de cambio de uso del suelo se calcularon mediante la fórmula de Palacio-Prieto et al. (2004): $\mathrm{Td}=$ [(S2 / S1) (1 / n) -1] * 100, donde: Td = Tasa de cambio anual (\%), S1 = Área cubierta al inicio del periodo (ha), S2 = Área cubierta al final del periodo (ha), y $n=$ Número de años del periodo.

\section{Modelación con Cadenas de Markov}

Con el módulo Markov de IDRISI Selva se creó una matriz de probabilidad de transición. El método consistió en cruzar mapas de uso del suelo en dos periodos de tiempo, con un margen de error del $15 \%$, para crear una matriz de probabilidad de cambio en un tercer periodo (Eastman, 2012). Los resultados de la ejecución de este comando fueron: una matriz de probabilidad de transición, una matriz de áreas de transición, y una colección de mapas que representan las áreas de adecuación/aptitud para las seis categorías de uso del suelo. La matriz creada fue 2000-2010 (Proyección 2030) (Figura 4).

\section{Modelación con Autómatas Celulares}

Con el módulo CA-Markov de IDRISI Selva se construyeron escenarios espaciales a partir de la combinación de un mapa de uso de suelo 2010 (Figura 3), la matriz de áreas de cambio y la colección de imágenes de áreas de adecuación/aptitud construidas en Markov (2030). El modelo se corrió con 12 interacciones y un filtro de contigüidad de $5 \times 5$ píxeles para predecir cambios celulares significativos (Eastman 2012). El resultado fue la generación de un escenario espacial 2030 (Figura 4), que fue comparado el mapa de uso de suelo 2010 (Figura 3), mediante el MCT y se obtuvieron las pérdidas y ganancias (2010-2030).

\section{Resultados}

\section{Modelación de cambios 2000-2010}

Para este primer aspecto se detecta que en el año 2000 , el uso agrícola ocupaba el 30,5\% de la superficie, de la misma forma el pastizal representaba 47,2\% (Tabla $1)$, siendo las dos categorías con mayor dominio como consecuencia de la deforestación en las últimas cuatro décadas en Tabasco, principalmente en la región Chontalpa (Sánchez-Munguía, 2005; Zavala y Castillo, 2007). Como se aprecia en la Tabla 1 , el uso urbano representaba el $1 \%$ del territorio, sin embargo, el cambio de uso del suelo se mantenía en aumento debido al crecimiento de la población y las prácticas agropecuarias en la región Chontalpa (Pinkus-Rendón y ContrerasSánchez, 2012). La vegetación hidrófita y el manglar ocupaban menos espacio $(7,4 \%$ y $4,9 \%)$, mientras que los cuerpos de agua representaban el 9,1\% (Tabla 1).

Tabla 1

Cuantificación del cambio de uso del suelo 2000-2010

\begin{tabular}{|c|c|c|c|c|c|c|c|c|c|}
\hline$\%$ & 2000 & $\%$ & 2010 & $\%$ & GANANCIAS & $\%$ & PÉRDIDAS & $\%$ & $\begin{array}{c}\text { TASAS DE } \\
\text { CAMBIO } \\
\%\end{array}$ \\
\hline Agricultura & 23.612 & $30,5 \%$ & 26.104 & $33,7 \%$ & 8.391 & $6,1 \%$ & 5.899 & $4,3 \%$ & $1 \%$ \\
\hline Pastizal & 36.575 & $47,2 \%$ & 33.445 & $43,1 \%$ & 7.474 & $5,4 \%$ & 10.604 & $7,7 \%$ & $0,9 \%$ \\
\hline Vegetación hidrófita & 5.705 & $7,4 \%$ & 5.074 & $6,5 \%$ & 2.568 & $1,9 \%$ & 3.198 & $2,3 \%$ & $1,2 \%$ \\
\hline Urbano & 807 & $1 \%$ & 1.518 & $2 \%$ & 810 & $0,6 \%$ & 0 & $0,1 \%$ & $6,1 \%$ \\
\hline Cuerpos de agua & 7.037 & $9,1 \%$ & 6.577 & $8,5 \%$ & 1.182 & $0,7 \%$ & 1.642 & $1,2 \%$ & $0,7 \%$ \\
\hline
\end{tabular}

Fuente: Elaboración propia (MCT). 
En el 2010, las superficies de uso agrícola y pastizal ocuparon $33,7 \%$ y $43,1 \%$ del territorio, el uso agrícola presentó una elevada tasa de crecimiento de $1 \%$, mientras que el pastizal presentó una tasa de pérdida de $0,9 \%$. A pesar del descenso del pastizal, este uso junto con el agrícola, continuaron dominando y fragmentado el paisaje natural de Comalcalco. De la misma forma, el uso urbano aumentó el doble de su superficie, ocupando el $2 \%$ del territorio, lo que representa la mayor tasa de aumento $(6,1 \%)$ durante el periodo de estudio. En ese sentido, ante el continuo aumento de los usos antropogénicos en el municipio, la vegetación hidrófita y los cuerpos de agua perdieron elevadas superficies, llegando al 6,5\% y el $8,5 \%$ del municipio, mientras que el manglar aumentó superficies importantes, ocupando el $6,2 \%$ del territorio. Las tasas de cambio que expresaron la pérdida de la vegetación hidrófita y los cuerpos de agua fueron elevadas $(1,2 \%)$ y $(0,7 \%)$ respectivamente, mientras que el manglar presentó una tasa de crecimiento del 2,4\%.

\section{Pérdidas y ganancias (2000-2010)}

Para entender la dinámica de las coberturas naturales y los usos antropogénicos, el MCT cuantificó que la agricultura obtuvo elevadas ganancias de 8.391 ha, sin embargo, también presentó pérdidas de 5.899 ha, mientras que el pastizal presentó ganancias de 7.474 ha, ante grandes pérdidas de 10.604 ha (Tabla 1). El uso urbano solo presentó ganancias de 810 ha, y nulas pérdidas, demostrando que la infraestructura artificial difícilmente volvería a convertirse en cobertura natural. Los cuerpos de agua ganaron 1.182 ha, pero también perdieron alrededor de 1.642 ha, mientras que la vegetación hidrófita obtuvo ganancias de 2.568 ha, pero fueron mayores sus pérdidas de 3.198 ha. También se detecta que la cobertura manglar presentó importantes ganancias de 1.646 ha ante pérdidas de 628 ha (Tabla 1 ).

\section{Contribuciones entre categorías (2000-2010)}

En relación con las contribuciones más importantes entre categorías, el pastizal, la vegetación hidrófita y los cuerpos de agua perdieron superficies de 2.758, 95 y 103 ha para el crecimiento de la agricultura, mientras que el manglar y uso urbano absorbieron 235 y 228 ha a la agricultura (Tabla 2). La vegetación hidrófita cedió superficies de 234 y 281 ha para el crecimiento del pastizal y uso urbano. El pastizal perdió importantes superficies de 483 ha para el crecimiento del uso urbano y perdió 98 ha para convertirse en cuerpos de agua. Es importante mencionar que el manglar desplazó 737 ha de cuerpos de agua (Tabla 2).

Tabla 2

Contribuciones entre categorías 2000-2010. Medidas en hectáreas.

\begin{tabular}{lccclc}
\hline Agricultura & \multicolumn{2}{l}{ Vegetación hidrófita } & \multicolumn{2}{l}{ Urbano } & \\
\hline Agricultura & 0 & Agricultura & -95 & Agricultura & 228 \\
\hline Pastizal & 2.758 & Pastizal & -234 & Pastizal & 483 \\
\hline $\begin{array}{l}\text { Vegetación } \\
\text { hidrófita }\end{array}$ & 95 & $\begin{array}{l}\text { Vegetación } \\
\text { hidrófita }\end{array}$ & 0 & $\begin{array}{l}\text { Vegetación } \\
\text { hidrófita }\end{array}$ & 0 \\
\hline Manglar & -235 & Manglar & -21 & Manglar & 0 \\
\hline $\begin{array}{l}\text { Urbano } \\
\text { Cuerpos de } \\
\text { agua }\end{array}$ & -228 & Urbano & 0 & Urbano & 0 \\
\hline
\end{tabular}

\begin{tabular}{lclclc} 
Pastizal & \multicolumn{2}{l}{ Manglar } & \multicolumn{3}{l}{ Cuerpos de agua } \\
\hline Agricultura & -2.758 & Agricultura & 235 & Agricultura & -103 \\
\hline Pastizal & 0 & Pastizal & 25 & Pastizal & 98 \\
\hline $\begin{array}{l}\text { Vegetación } \\
\text { hidrófita }\end{array}$ & 234 & $\begin{array}{l}\text { Vegetación } \\
\text { hidrófita }\end{array}$ & 21 & $\begin{array}{l}\text { Vegetación } \\
\text { hidrófita }\end{array}$ & 281 \\
\hline Manglar & -25 & Manglar & 0 & Manglar & -737 \\
\hline $\begin{array}{l}\text { Urbano } \\
\text { Cuerpos de }\end{array}$ & -483 & Urbano & 0 & Urbano & 0 \\
agua & -98 & $\begin{array}{l}\text { Cuerpos de } \\
\text { agua }\end{array}$ & 737 & $\begin{array}{l}\text { Cuerpos de } \\
\text { agua }\end{array}$ & 0 \\
\hline
\end{tabular}

Fuente: Elaboración propia (MCT).

\section{Matriz de probabilidad de cambios 2030 (Markov)}

La matriz de cambios (2030) (Tabla 3) demostró que superficies agrícolas presentaron una elevada probabilidad de 0.31 para transformarse en superficies de pastizal y 0.16 para convertirse en vegetación hidrófita. De la misma forma, superficies de pastizal obtuvieron 0.31 de probabilidad para transformarse en uso agrícola y presentaron una importante probabilidad de 0.40 para su transformación a vegetación hidrófita. Como es de esperar, áreas de pastizal presentaron probabilidad de 0.16 paras transformarse a urbano, de la misma forma presentó una probabilidad de 0.10 para convertirse en cuerpos de agua. Las coberturas de vegetación hidrófita presentaron probabilidades de 0.10 para ser remplazadas por manglar. Mientras que superficies del manglar se transformará en cuerpos de agua con probabilidad de 0.19 . 
Tabla 3

Matriz de probabilidades de cambio 2030

\begin{tabular}{|c|c|c|c|c|c|c|}
\hline & $\begin{array}{c}\text { Agricultura } \\
\%\end{array}$ & $\begin{array}{c}\text { Pastizal } \\
\%\end{array}$ & $\begin{array}{c}\text { Vegetación hidrófita } \\
\%\end{array}$ & $\begin{array}{c}\text { Manglar } \\
\%\end{array}$ & $\begin{array}{c}\text { Urbano } \\
\%\end{array}$ & $\begin{array}{c}\text { Cuerpos de agua } \\
\%\end{array}$ \\
\hline Agricultura & 60 & 31 & 2 & 2 & 2 & 0 \\
\hline Pastizal & 31 & 56 & 5 & 1 & 3 & 2 \\
\hline Vegetación hidrófita & 16 & 40 & 21 & 8 & 0 & 12 \\
\hline Manglar & 3 & 8 & 10 & 70 & 0 & 6 \\
\hline Urbano & 6 & 16 & 0 & 0 & 77 & 0 \\
\hline Cuerpos de agua & 4 & 10 & 5 & 19 & 00 & 59 \\
\hline
\end{tabular}

Fuente: Elaboración propia (MARKOV).

\section{Proyección espacial 2030}

En comparación con el uso del suelo del 2010, la proyección espacial del 2030 (Figura 4) muestra un escenario en donde aumenta la dinámica del paisaje (Tabla 4). Se observa que el uso agrícola aumentó de superficie, representando el 35,8\% (27.781 ha), mientras que el pastizal continuó presentando elevadas disminuciones hasta alcanzar el 39,4\% (30.532 ha) del territorio; de la misma forma, el uso urbano aumentó de superficie, ocupando el 3,4\% (2.631 ha) (Tabla 4). En general, el escenario 2030 muestra el inminente dominio antropogénico sobre el paisaje natural. En relación con las coberturas naturales, se detecta que la vegetación hidrófita y los cuerpos de agua presentaron importantes disminuciones de superficie, representando el 5,9\% (4.604 ha) y 7,5\% (5.816 ha) respectivamente (Tabla 4). Sin embargo, el manglar siguió aumentando espacio, llegando a un 7,5\% (6.155 ha) del territorio (Tabla 4).

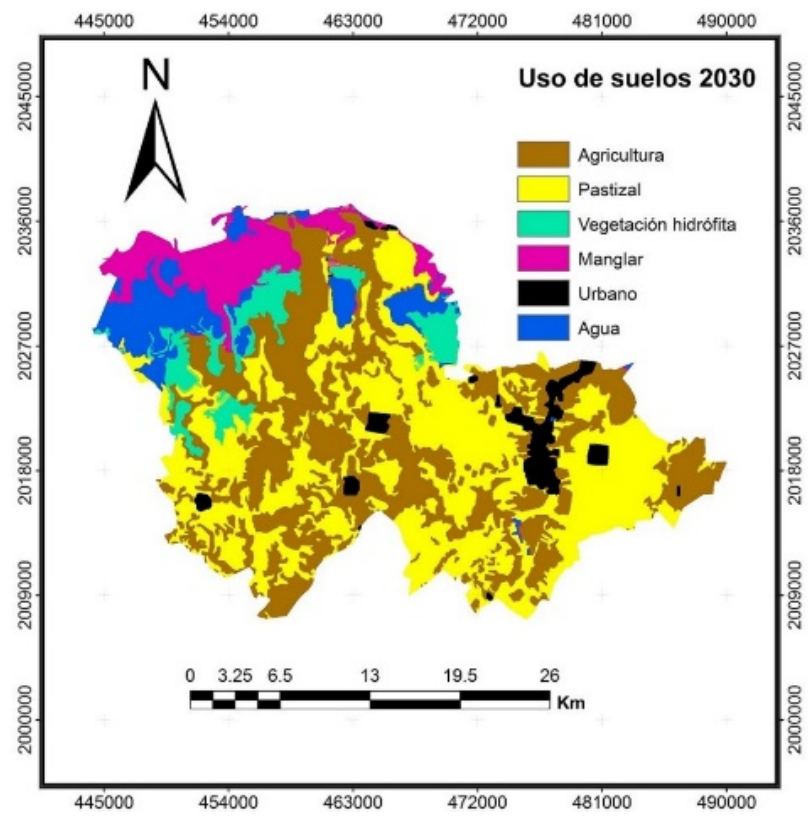

Figura 4. Mapa de uso de suelos en Comalcalco (2030).

Fuente: Elaboración propia a partir de CA-MARKOV 
Tabla 4

Cambio de uso del suelo 2010-2030

\begin{tabular}{|c|c|c|c|c|c|c|c|c|}
\hline Categoría & 2010 & $\%$ & 2030 & $\%$ & Ganancias & $\%$ & Pérdidas & $\%$ \\
\hline Agricultura & 26.104 & 33.7 & 27.781 & 35.8 & 2.425 & 1.8 & 745 & 0.5 \\
\hline Pastizal & 33.445 & 43.1 & 30.532 & 39.4 & 137 & 0.1 & 3.050 & 2.2 \\
\hline Vegetación hidrófita & 5.074 & 6.5 & 4.604 & 5.9 & 75 & 0.05 & 544 & 0.4 \\
\hline Manglar & 4.801 & 6.2 & 6.155 & 7.9 & 1.415 & 1.03 & 61 & 0.04 \\
\hline Urbano & 1.518 & 2 & 2.631 & 3.4 & 1.110 & 0.8 & 0 & 0 \\
\hline Cuerpos de agua & 6.577 & 8.5 & 5.816 & 7.5 & 288 & 0.2 & 1.049 & 0.7 \\
\hline
\end{tabular}

Fuente: Elaboración propia (MCT).

\section{Discusión}

La modelación del cambio de uso del suelo en Comalcalco, Tabasco, México, detectó con gran precisión, las pérdidas de la vegetación hidrófita y cuerpos de agua, ante el crecimiento de los usos antropogénicos como la agricultura y la expansión de la zona urbana que han provocado la dinámica del paisaje natural. Así mismo, se detectaron disminuciones del pastizal de origen pecuario, las que también contribuyeron a la dinámica; sobresale al respecto el aumento importante del manglar en las costas, siendo un indicador del cambio climático por el ascenso del nivel el mar (Amatya et al., 2009; Sun \& Lockaby, 2012). En general el territorio se está expandiendo incontrolablemente ante un deficiente desarrollo sostenible, en tanto que el paisaje natural está totalmente fragmentado con efectos ambientales, lo cual es común en países latinoamericanos (Bähr y Borsdorf, 2005). Estos cambios en el paisaje se relacionan con los reglamentos de usos del suelo, la economía regional, los movimientos de población debido a programas de desarrollo económico, la demanda de productos agrícolas mexicanos y el entorno político (Linard, Tatem, \& Gilbert, 2013). Al mismo tiempo el territorio presenta la influencia del relieve plano, la escasa pendiente y las carreteras que facilitan el establecimiento de nuevos centros de población (Gutiérrez, Condeço-Melhorado \& Martín, 2010; Kolb, Mas \& Galicia, 2013).

En Comalcalco, la mayor superficie alterada por las actividades agropecuarias y el crecimiento urbano se ha dado en la parte sur, mientras que en el norte se han dado en menor proporción, debido a factores naturales como la recuperación de manglares en los humedales (Ramos-Reyes, Sánchez-Hernández y Gama-Campillo, 2016). Así mismo, se comprobó durante el 2010 un mayor número de zonas urbanas (10 núcleos poblacionales) cercanos a los límites de los municipios de H. Cárdenas y Jalpa de Méndez, destacando la ampliación de la cabecera municipal de áreas que en el pasado fueron de uso agrícola y ganadero (Ramos-Reyes et al., 2016). Esta tendencia del crecimiento urbano coincide con el grado de cambios espaciales alcanzado en distintas regiones de México, los que no han contribuido con un incremento substancial en el desarrollo del país. Por el contrario, gran parte de los problemas sociales, como la segregación y desigualdad económica y el deterioro ambiental, la deforestación de los bosques, la fragmentación de los ecosistemas y contaminación de humedales en México, se han originado por el desarrollo urbano y periurbano no planificado (Garza, 2010; MacGregor-Forsy y Ortega-Álvarez, 2013).

A nivel estatal, el mayor deterioro de los ecosistemas se dio entre los años 60 y 70, con la ejecución de los programas de desarrollo económico y agropecuarios que impulsó a la ganadería extensiva para abastecer al mercado mediante el Plan Chontalpa (1965-1976) y el Programa de Desarrollo Rural Integrado para el Trópico Húmedo, que en conjunto con el auge petrolero de los años 70 (Arrieta, 1994; Capdepont-Ballina y Marín-Olán, 2014; Flores-Santiago, 1987), mejoraron las condiciones de vida de las poblaciones de la región. Es así como se crearon nuevas zonas urbanas, se construyeron nuevos caminos y carreteras federales y se desarrolló la infraestructura hidráulica para controlar las inundaciones, los que acarrearon grandes tasas de deforestación de la selva (Arrieta, 1994; Flores-Santiago, 1987; Murillo y Martínez, 2010; Pinkus-Rendón y Contreras-Sánchez, 2012; Sánchez-Munguía, 2005; Zavala y Castillo, 2007). Principalmente se crearon 
infraestructuras que favorecieron al desarrollo de Tabasco, provocando que el crecimiento poblacional y urbano aumentara en los municipios de H. Cárdenas, Comalcalco, Centla, Paraíso y Macuspana (Pinkus-Rendón y Contreras-Sánchez, 2012).

El análisis espacial señala que el uso agrícola fue el factor primordial de la pérdida de pastizal, vegetación hidrófita y cuerpos de agua, y se estima que su tasa de aumento fue muy elevada, un $1 \%$. Esto es debido a las políticas de apoyo al campo mexicano, las que también desempeñan un papel importante en el deterioro del paisaje natural en Tabasco. Por ejemplo, el Programa de apoyos directo al campo, PROCAMPO, es una iniciativa federal destinada a los pequeños y medianos agricultores, y tiene como objetivos: fomentar la reconversión productiva hacia actividades de mayor rentabilidad, compensar subsidios que otros países otorgan a sus productores, estimular la organización de los productores, incrementar la competitividad de las cadenas productivas y frenar la degradación del medio ambiente (Zarazúa-Escobar, Almaguer-Vargas y OcampoLedesma, 2011). Sin embargo, el aumento en el número de hectáreas de labor para el establecimiento de maíz, frijol, plátano, papaya, caña de azúcar, pepino y calabaza, con el objetivo de recibir más apoyos económicos por parte de PROCAMPO, propició que los productores transformaran los cuerpos de agua, la vegetación hidrófita y los pastizales en superficies de cultivos agrícolas, acelerando los procesos de deforestación y dejando en total desventaja al objetivo de frenar la degradación ambiental (Klepeis \& Vance, 2003; Kolb et al., 2013; Schmook y Vance, 2009).

Otro apoyo que influyó en el crecimiento de la agricultura en Comalcalco fue el Programa de Apoyo a la Cadena Productiva de Productores de maíz y frijol, y el Proyecto Estratégico de seguridad Alimentaria, PESA, los cuales impulsaron la producción de cultivos de maíz y frijol, naranja, mango, coco, plátano, papaya, caña de azúcar, maíz, frijol, pepino y calabaza (Baca del Moral, 2014). Cabe destacar que el cacao (Figura 5) es el principal cultivo y la fuente de ingreso más importante del sector agrícola, representando más del 30\% de los cambios de uso del suelo en el municipio a principio del siglo XXI (Ramos-Reyes, Palma-López, Ortiz-Solorio, OrtizGarcía y Díaz-Padilla, 2004).
A nivel estatal, el crecimiento del pastizal en las últimas dos décadas fue debido a los créditos de apoyo al establecimiento de ganado bovino (PROGAN) (Figura 5). Este programa brinda varios créditos en zonas pecuarias para el mejoramiento de las instalaciones y subsidios para compra y venta de ganado. Esto ocasionó, al igual que PROCAMPO, que aumentaran las hectáreas de pastizal para compra y manejo de cabezas de ganado (Kolb et al., 2013). A pesar del crecimiento del pastizal ante la deforestación de la vegetación arbórea en Tabasco para el establecimiento de ganado bovino, en Comalcalco se detectó una notable disminución del pastizal de 3.050 ha entre 2000-2010, debido a otros factores que provocaron una contracción de la actividad pecuaria, entre los que se destacan: la ganadería extensiva con bajo nivel de tecnificación, la estacionalidad, el impacto del estiaje y las grandes inundaciones en Tabasco, los que en su conjunto provocaron una baja rentabilidad de la actividad; finalmente esto terminó afectando principalmente a los pequeños productores (Castro, Tewolde y Toral, 2002). Otro factor que también afectó a la reducción de la actividad fue la reducción de los canales de comercialización de la carne en el estado y de la apertura del mercado de ganado hacia el norte del país y los Estados Unidos (Ramos-Reyes et al., 2004).

Por su parte, los cuerpos de agua de las regiones Chontalpa, Pantanos y Centro, representan grandes extensiones de humedales y alcanzan el $15,6 \%$ del estado de Tabasco (Barba-Macías, Rangel-Mendoza y RamosReyes, 2006). Sin embargo, en este estudio se detecta que los cuerpos de agua están siendo alterados por el crecimiento del pastizal, a pesar de que son ecosistemas productivos, siendo grandes fuentes de diversidad biológica, debido a que son reservorios de agua vital para los organismos, y también son responsables de alta productividad primaria que beneficia a las cadenas tróficas (Smith-Guerra y Romero-Aravena, 2009). Cuando los cuerpos de agua son deteriorados, existen alternativas de restauración ecológica, sin embargo, se trata de técnicas costosas que requieren de una amplio conocimiento detallado del ecosistema para su manejo adaptativo y, sobre todo, precisan ser insertadas en las políticas públicas (Espinoza-Tenorio, Zepeda-Domínguez, Núñez-Gómez, Mendoza-Carranza y Barba-Macías, 2015; 
Rojas, Sepúlveda-Zúñiga, Barbosa, Rojas, y Martínez, 2015; Zhang y Shao, 2013).

Entre las coberturas naturales deterioradas se encuentra la vegetación hidrófita (Figura 5), reconocidas como vegetación acuática y subacuática por Rzedowsky (1978). Por su parte, Miranda y Hernández (1963) las dividen en tular, popal y carrizal, mientras que West, Psuty y Thom (1976) identifican dos comunidades: popalería y plantas acuáticas y flotantes. En este estudio se detectó que estas coberturas perdieron superficies importantes para el crecimiento del pastizal y uso urbano, con una tasa de pérdida elevada de $1,2 \%$, la que coincide con los estudios a nivel nacional que señalan que la permanencia de la vegetación hidrófita en las costas mexicanas es muy preocupante, ya que su tasa de pérdidas se situó en 4,6\% durante 2000-2007 (RoseteVergés et al., 2014).

Otro ecosistema de gran relevancia es el manglar ubicado en las costas de Tabasco (Figura 5). Los manglares de la región constituyen especies de Rhizophora mangle, Avicennia germinans, Laguncularia racemosa y Conocarpus erectus (Novelo, 2006; RoseteVergés et al., 2014). En este estudio se detectó una tasa de crecimiento del manglar en 2,4\%, debido a que creció en áreas que pertenecían al uso agrícola y a los cuerpos de agua en la zona norte del municipio, esto se comprueba con las ganancias de 1.646 ha, siendo similar a lo reportado por Guerra-Martínez y Ochoa-Gaona (2006) quienes detectaron que durante 1990-2000, en la Reserva de la Biosfera, Pantanos de Centla, el manglar aumentó importantes superficies de 808 ha. Esto se debe a que las mareas influyen para que el suelo se convierta en un sustrato adecuado para el crecimiento del manglar, debido a la penetración del agua salada (Sánchez, Ulloa, y Ricardo, 1998), siendo evidencia del ascenso del nivel del mar, debido al cambio climático (Sun \& Lockaby, 2012). Esto demuestra que los manglares acomodan su distribución ante la probabilidad de nuevas magnitudes de parámetros ambientales, lo cual permite fundamentar que los manglares son ecosistemas centinela, frente a los efectos del cambio climático en el Golfo de México (Yáñez-Arancibia, Day, Twilley y Day, 2014).

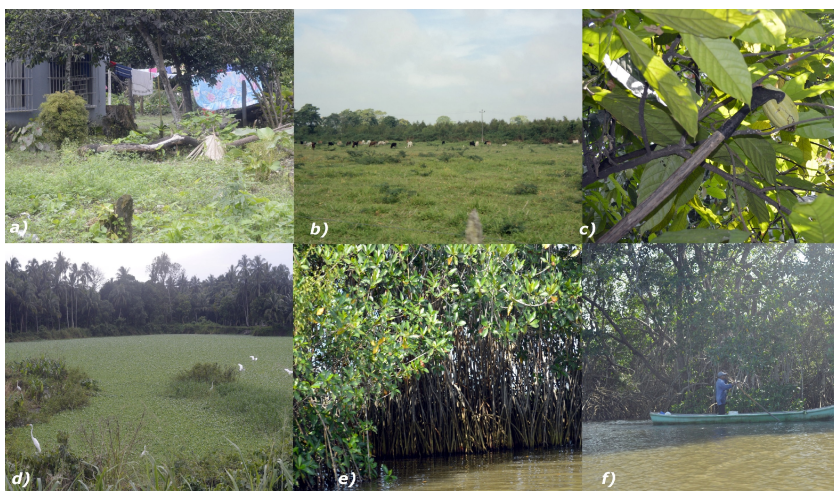

Figura 5. Imágenes de usos antropogénicos y coberturas naturales en Comalcalco, Tabasco: a) Zona urbana, b) Pastizal de uso pecuario, c) Cultivo de cacao, d) Vegetación hidrófita e) Manglar y f) Cuerpos de agua.

Fuente: Elaboración Propia (trabajo de campo).

Sin embargo, Landgrave y Moreno-Casasola (2012) cuantificaron que durante 1979-2008 en Tabasco se han perdido alrededor de 912.942 ha de manglares equivalentes al $60 \%$ del territorio. De la misma forma, Ramírez-García, López-Blanco y Ocana (1998) detectaron una tasa de cambio de uso del suelo del manglar de 1,4\% (1970-1993). Esto significa que a pesar de que los manglares son ecosistemas que proveen servicios ecológicos y económicos a la población (Yáñez-Arancibia et al., 2014), la falta de un reconocimiento real de su importancia ambiental, económica y social ha provocado su deterioro (Hirales-Cota et al., 2010). Los factores de deforestación más frecuentes del manglar son el establecimiento de pastizales, la construcción de caminos y la tala para la construcción de casas, incendios forestales, límites de propiedad, tala para la construcción de caminos e impactos de huracanes (Guerra-Martínez y Ochoa-Gaona, 2006; Hernández-Melchor, Rosado, Sánchez, y Hernández, 2016; Hirales-Cota et al., 2010). De la misma forma, la actividad petrolera ha generado impactos negativos en el manglar debido a la contaminación de los humedales y la degradación del suelo (Olguín, Hernández y Sánchez-Galván, 2007).

Este análisis lleva a la reflexión de que, en México, el desarrollo sustentable debe implicar una planificación que incluya la creación de áreas naturales protegidas, tierras de trabajo para los habitantes dedicados al sector primario y la disponibilidad de infraestructura urbana en zonas no vulnerables, ante los estragos del cambio climático (Benítez, Pérez-Vázquez, Nava-Tablada, 
Equihua \& Álvarez-Palacios, 2012). El escenario de pérdida de las coberturas naturales en los próximos trece años representa una alerta para los responsables de diseñar y aplicar programas de planeación urbana enfocados a mitigar o revertir los efectos adversos en el sistema natural para beneficio de la población.

Esta tendencia adversa puede ser mitigada mediante el planeamiento basado en el diseño de un programa de regulación urbana incluyente, transdisciplinario, transparente y sustentable, que integre multiescalas espaciales y temporales, respete el marco normativo, actualice las reservas territoriales, promueva la conservación y creación de áreas naturales protegidas e incluya el ordenamiento territorial (Secretaría de Energía, Recursos Naturales y Protección Ambiental, SERNAPAN, 2013). Por ejemplo, el programa estatal de ordenamiento ecológico 2013-2018 (SERNAPAN, 2013), señala que es importante que, en el municipio de Comalcalco, principalmente en el noroeste, norte y noreste donde se ubican la vegetación hidrófita, cuerpos de agua y los crecientes manglares, sea planificado mediante el establecimiento de: (1) Área Natural Protegida (1,30\%), (3) Zona Conservación (17,47\%), y Área Prioritaria de Conservación (10,73\%). También define la creación de (4) Zona de Aprovechamiento (70,50\%), en el este, sureste, sur y suroeste, donde predominan la mayor parte de las actividades agrícolas, pecuarias y de uso urbano (Figura 6).

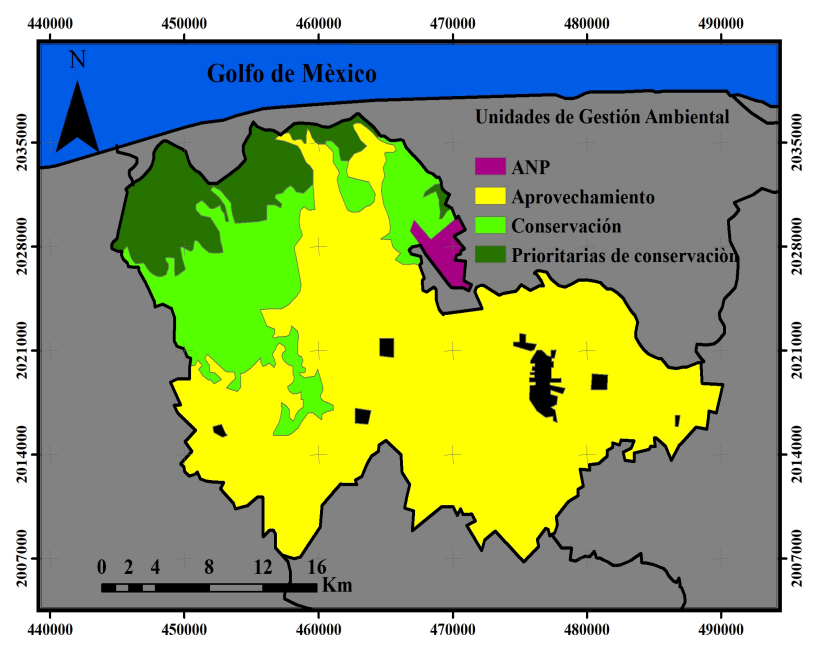

Figura 6. Unidades de Gestión Ambiental para la planeación ambiental de Comalcalco.

Fuente: SERNAPAN, 2013.
Sin embargo, la creación jurídica de zonas de conservación biológica en los municipios de México está aún pendiente de ser una realidad, debido a las inconsistencias legales y administrativas entre los programas de desarrollo urbano locales, estatales, así como entre las leyes locales y federales en materia ambiental, y por la insuficiencia de ingresos económicos en gran parte de la población en las localidades urbanas y suburbanas en México (Bazant, 2010; Orellana, Arenas, Marshall \& Rivera, 2016).

Se comprueba que el Modelador de Cambios en el Terreno, las Cadenas de Markov y los Autómatas Celulares detectaron con precisión la distribución de las coberturas naturales y usos antropogénicos, las probabilidades y la proyección espacial 2030. Por lo tanto, para evitar escenarios de deterioro ambiental. Jiménez-Moreno, González-Guillen, Escalona-Maurice, Valdez-Lazalde y Aguirre-Salado (2011), mencionaron que es imprescindible el empleo de uno o varios modelos de cambio de uso del suelo, para que las autoridades y planificadores del suelo puedan comprender el alcance de los cambios registrados, las causas y los riesgos que ello implica, para dar seguimiento al ordenamiento territorial en las zonas urbanas y agropecuarias de 


\section{Conclusiones}

El cambio de uso del suelo en Comalcalco, Tabasco, México, se modeló con base en el efecto provocado por el crecimiento de las actividades antropogénicas sobre las coberturas naturales. Se hizo un análisis espacial mediante el Modelador de Cambios en el Terreno (MCT) y se calcularon tasas de cambio. Los resultados indican que durante 2000-2010 se perdieron 8.391 ha de vegetación hidrófita y 1.048 ha de cuerpos de agua, ante el crecimiento acelerado de 8.391 ha de uso agrícola y 810 ha de la zona urbana. Así mismo, se reveló una pérdida de 3.050 ha de pastizal y el aumento de 1.415 ha de manglar. Las Cadenas de Markov y los Autómatas Celulares proyectaron un escenario (2030) en donde se pierden 544 ha de vegetación hidrófita, 1.048 ha de cuerpos de agua y 3.050 de pastizal, ante los aumentos de 2.425 ha de uso agrícola, 1.415 ha del manglar y 1.110 de la zona urbana.

Los resultados reafirman que el modelo de desarrollo territorial de Comalcalco ha omitido, en general, la conservación de los ecosistemas de humedales, en especial de los cuerpos de agua y vegetación hidrófita. Si continúa esta tendencia de cambio de uso del suelo, por el crecimiento de las actividades agropecuarias y centros urbanos mal planificado en el noroeste, norte y noreste

\section{Referencias}

Akinbobola, A. (2015). Land use/land cover change detection in some selected stations in Anambra State. Journal of Geography and Regional Planning, 8(1), 111. http://dx.doi.org/10.5897/JGRP2014.0468

Al-shalabi, M., Billa, L., Pradhan, B., Mansor, S., \& AlSharif, A. A. (2013). Modelling urban growth evolution and land-use changes using GIS based cellular automata and SLEUTH models: The case of Sana'a metropolitan city, Yemen. Environmental earth sciences, 70(1), 425-437. Recuperado de: https://link.springer.com/article/10.1007/s12665$\underline{012-2137-6}$ del municipio, se pueden perder las coberturas de manglares, vegetación hidrófita y cuerpos de agua aún conservados en los próximos trece años. Por lo tanto, para evitar escenarios de deterioro ambiental mostrados en la proyección espacial (2030), resalta la importancia de proteger la vegetación hidrófita, los cuerpos de agua y las crecientes superficies de manglar mediante una planificación ambiental que establezca formalmente zonificaciones definidas como: Área Natural Protegida, Conservación, Prioritaria de Conservación y Aprovechamiento, como lo señala el Programa de Ordenamiento Ecológico.

Se demuestra que la integración de modelos geomáticos para estudiar el cambio de uso del suelo y proyecciones futuras es una herramienta precisa para simular la dinámica interna del paisaje y obtener probabilidades de cambios. La aportación de este estudio es adecuada para la planeación ambiental, a través de instrumentos como el ordenamiento ecológico territorial, planes de desarrollo urbano y agropecuario, y en la creación de políticas, para el óptimo manejo de los recursos naturales, proporcionando oportunidades a los planificadores para hacer frente a futuros problemas ambientales en el sureste mexicano [B]

Amatya, D. M., Skaggs, R. W., \& Trettin, C. C. (2009). Advancing the Science of Forest Hydrology. A challenge to agricultural and biological engineers. Resource Magazine, 16(5), 10-11. Recuperado de https://elibrary.asabe.org/abstract.asp?aid=29315

Arrieta, F. P. (1994). La integración social de la Chontalpa: un análisis regional en el trópico mexicano. México DF: Gernika.

Baca del Moral, J. (2014). El Programa Especial para la Seguridad Alimentaria (PESA), nueva forma de extensionismo, en México. Spanish Journal of Rural Development, 5(3), 33-42. http://dx.doi.org/10.5261/2014.GEN3.04 
Bähr, J. y Borsdorf, A. (2005). La ciudad latinoamericana. La construcción de un modelo. Vigencia y perspectivas. Urbe. Revista de ciudad, urbanismo y paisaje (Lima), 2(2), 207-222. Recuperado de http://www.guzlopeditoras.com/web des/arquit01/pld0472.pdf

Barba-Macías, E., Rangel-Mendoza, J., y Ramos-Reyes, R. (2006). Clasificación de los humedales de Tabasco mediante sistemas de información geográfica. Ecosistemas y Recursos Agropecuarios, 22(2). Recuperado de http://era.ujat.mx/index.php/rera/article/view/313/2 $\underline{50}$

Bazant, S. (2010). Expansión urbana incontrolada y paradigmas de la planeación urbana. espacio abierto, 19(3), 475-503. Recuperado de http://produccioncientificaluz.org/index.php/espacio /article/view/1405/1407

Benítez, G., Pérez-Vázquez, A., Nava-Tablada, M., Equihua, M., \& Álvarez-Palacios, J. L. (2012). Urban expansion and the environmental effects of informal settlements on the outskirts of Xalapa city, Veracruz, Mexico. Environment and Urbanization, 24(1), 149166. https://doi.org/10.1177/0956247812437520

Berberoğlu, S., Akın, A., \& Clarke, K. C. (2016). Cellular automata modelling approaches to forecast urban growth for Adana, Turkey: A comparative approach. Landscape and Urban Planning, 153, 11-27. https://doi.org/10.1016/j.landurbplan.2016.04.017

Brena, J., Castillo, C., y Wagner, A. (2016). Metodología para la delimitación y caracterización de humedales en escalas 1:50 000 y 1:20 000. Tecnología y ciencias del agua, 7(2), 85-98. Recuperado de http://www.scielo.org.mx/pdf/tca/v7n2/2007-2422tca-7-02-00085.pdf

Castro, H. G., Tewolde, A. M., y Toral, J. N. (2002). Análisis de los sistemas ganaderos de doble propósito en el centro de Chiapas, México. Archivos Latinoamericanos de Producción Animal, 10(17), 5183.

https://www.researchgate.net/publication/26624535 8
Capdepont-Ballina, J. L. y Marín-Olán, P. (2014). La economía de Tabasco y su impacto en el crecimiento urbano de la ciudad de Villahermosa (1960-2010). LiminaR, 12(1), 144-160. Recuperado de http://www.scielo.org.mx/scielo.php?pid=S16658027 $\underline{2014000100010 \& \text { script }=\text { sci } \_ \text {arttext\&tlng=en }}$

Challenger, A., Dirzo, R., López, J. C., Mendoza, E., LiraNoriega, A., y Cruz, I. (2009). Factores de cambio y estado de la biodiversidad. Capital natural de México, 2, 37-73.

Clarke, K. C. \& Gaydos, L. J. (1998). Loose-coupling a cellular automaton model and GIS: Long-term urban growth prediction for San Francisco and Washington/Baltimore. International Journal of Geographical Information Science, 12(7), 699-714. http://dx.doi.org/10.1080/136588198241617

Eastman, J. R. (2012). IDRISI Selva GIS and image processing software version 17.0. Massachusetts, USA: Clark Labs.

Espinoza-Tenorio, A., Zepeda-Domínguez, J. A., NúñezGómez, J. C., Mendoza-Carranza, M., y Barba-Macías, E. (2015). ¿De la intuición al conocimiento científico? Publicaciones sobre las lagunas costeras de Tabasco, México. Interciencia, 40(7), 448-456. Recuperado de http://www.redalyc.org/html/339/33940000003/

Flores-Santiago, A. (1987). La modernización de la agricultura en el trópico húmedo mexicano: veinte años de experiencia en la Chontalpa, Tabasco. Revista de Geografía Agrícola, 13-14(2), 105-114. Recuperado de:

http://www.chapingo.mx/revistas/geografia/conteni do.php?id_articulo $=1735$ ? id_revistas $=4$ ? id_revista_n $\underline{\text { umero }=174}$

Garza, G. (2010). La transformación urbana de México, 1970-2020. En G. Garza y M. Schteingart (Eds.), Los grandes problemas de México. Desarrollo urbano y regional (pp. 31-86). Ciudad de México: El Colegio de México AC.

Godfray, H. C. J., Beddington, J. R., Crute, I. R., Haddad, L., Lawrence, D., Muir, J. F., \& Toulmin, C. (2010). Food security: The challenge of feeding 9 billion people. Science, 327(5967), 812-818. http://dx.doi.org/10.1126/science. 1185383 
Goldewijk, K. K., Beusen, A., Drecht, G. V., \& de Vos, M. (2011). The HYDE 3.1 spatially explicit database of human induced global land-use change over the past 12,000 years. Global Ecology and Biogeography 20(1), 73-86. $\quad$ http://dx.doi.org/10.1111/j.14668238.2010.00587.x

Guerra Martínez, V. y Ochoa Gaona, S. (2006). Evaluación espaciotemporal de la vegetación y uso del suelo en la Reserva de la Biosfera Pantanos de Centla, Tabasco (1990-2000). Investigaciones Geográficas, 59, 7-25. Recuperado de

http://www.scielo.org.mx/scielo.php?pid=S0188$46112006000100002 \&$ script $=$ sci_arttext\&tlng=en

Gutiérrez, J., Condeço-Melhorado, A., \& Martín, J. C. (2010). Using accessibility indicators and GIS to assess spatial spillovers of transport infrastructure investment. Journal of transport geography, 18(1), 141-152.

https://doi.org/10.1016/j.jtrangeo.2008.12.003

Hernández-Melchor, G. I., Rosado, O. R., Sánchez, Á. S., y Hernández, J. I. V. (2016). Cambios de uso del suelo en manglares de la costa de Tabasco. Revista Mexicana de Ciencias Agrícolas, 2757-2767. Recuperado de http://www.redalyc.org/pdf/2631/263144474009.pdf

Hirales-Cota, M., Espinoza-Avalos, J., Schmook, B., RuizLuna, A., y Ramos-Reyes, R. (2010). Agentes de deforestación de manglar en Mahahual-Xcalak, Quintana Roo, sureste de México. Ciencias marinas, 36(2), 147-159. Recuperado de http://www.scielo.org.mx/scielo.php?script=sci_artte xt\&pid=S0185-38802010000200004

Instituto Nacional de Estadística, Geografía e Informática, INEGI. (2006). Síntesis geográfica y anexo Cartográfico del Estado de Tabasco. Aguascalientes, México: INEGI.

Jiménez-Moreno, M. J., González-Guillen, M. D. J., Escalona-Maurice, M., Valdez-Lazalde, J. R., y AguirreSalado, C. A. (2011). Comparación de métodos espaciales para detectar cambios en el uso del suelo urbano. Revista Chapingo Serie Ciencias Gorestales y del Ambiente, 17(3), 389-406. http://dx.doi.org/10.5154/r.rchscfa.2010.04.020
Klepeis, P. \& Vance, C. (2003). Neoliberal policy and deforestation in southeastern Mexico: An assessment of the PROCAMPO program. Economic Geography, 79(3), 221-240.

Klanderud, K., Mbolatiana, H. Z. H., Vololomboahangy, M. N., Radimbison, M. A., Roger, E., Totland, Ø., \& Rajeriarison, C. (2010). Recovery of plant species richness and composition after slash-and-burn agriculture in a tropical rainforest in Madagascar. Biodiversity and Conservation, 19(1), 187-204. http://dx.doi.org/10.1007/s10531-009-9714-3

Kolb, M., Mas, J. F., \& Galicia, L. (2013). Evaluating drivers of land-use change and transition potential models in a complex landscape in Southern Mexico. International Journal of Geographical Information Science, 27(9), 1804-1827 http://dx.doi.org/10.1080/13658816.2013.770517

Landgrave, R., y Moreno-Casasola, P. (2012). Evaluación cuantitativa de la pérdida de humedales en México. Investigación Ambiental Ciencia y Política Pública, 4(1). Recuperado de http://www.revista.inecc.gob.mx/article/view/121\#. WVXtDIg1 IU

Lezama, J. L. (1987). Migración y petróleo en Tabasco. Estudios Demográficos y Urbanos, 2(2), 231-256. Recuperado

de: http://www.jstor.org/stable/40314407

Linard, C., Tatem, A. J., \& Gilbert, M. (2013). Modelling spatial patterns of urban growth in Africa. Applied Geography, 44 , 23-32. https://doi.org/10.1016/j.apgeog.2013.07.009

MacGregor-Forsy, F. I. y Ortega-Álvarez, Á. R. (2013). Ecología urbana: experiencias en América Latina. México, DF: INECOL.

Miller, R. W., Hauer, R. J., \& Werner, L. P. (2015). Urban forestry: Planning and managing urban green spaces. Long Groves: Waveland press.

Miranda, F. y E. Hernández-X. (1963). Los tipos de vegetación de México y su clasificación. Boletín de la Sociedad Botánica de México, 28, 29-179 http://dx.doi.org/10.17129/botsci.1084

Mitsch, W. J., Goseelink, J. G., \& Anderson, C. J. (2009). Wetland ecosystems. Hoboken, NJ: John Wiley \& Sons, Inc. 
Munteanu, C., Kuemmerle, T., Boltiziar, M., Butsic, V., Gimmi, U., Halada, L., \& Lieskovský, J. (2014). Forest and agricultural land change in the Carpathian region-a meta-analysis of long-term patterns and drivers of change. Land use policy, 38, 685-697. https://doi.org/10.1016/j.landusepol.2014.01.012

Murillo, L. D. y Martínez, R. J. (2010). Comunicación para el desarrollo en México: reflexiones sobre una experiencia en el trópico húmedo. Estudios sobre las Culturas Contemporáneas, 16(31), 201-225. Recuperado de http://www.culturascontemporaneas.com/contenido s/8\%20Testimonio\%20Murillo\%20pp\%20201-225.pdf

Newbold, T., Hudson, L. N., Hill, S. L., Contu, S., Lysenko, I., Senior, R. A., \& Day, J. (2015). Global effects of land use on local terrestrial biodiversity. Nature, 520(7545), 45-50.

http://dx.doi.org/10.1038/nature14324

Novelo, A. (2006). Plantas acuáticas de la Reserva de la Biosfera Pantanos de Centla. Villahermosa, México: Espacios Naturales y Desarrollo Sustentable, A.C

Olguín, E. J., Hernández, M. E., y Sánchez-Galván, G. (2007). Contaminación de manglares por hidrocarburos y estrategias de biorremediación, fitorremediación y restauración. Revista Internacional de Contaminación Ambiental, 23(3), 139-154. Recuperado de http://www.revistascca.unam.mx/rica/index.php/rica /article/view/21641/21640

Orellana, A., Arenas, F., Marshall, C., \& Rivera, A. (2016). Resistance to metropolitan institutionality and planning in Chile. Planning Practice and Research, 31, 435-451.

http://dx.doi.org/10.1080/02697459.2016.1196535

Organización de las Naciones Unidas para la Alimentación y la Agricultura, FAO. (2015). Evaluación de los recursos forestales mundiales 2015: FAO. Recuperado de http://www.fao.org/3/a-i4808s.pdf

Palacio-Prieto, J. L., Sánchez-Salazar, M. T., Casado, J. M., Propin, F. E., Delgado, C. J., Velázquez, M. A., y Camacho, R. C. G. (2004). Indicadores para la caracterización y el ordenamiento territorial. México: SEMARNAT. Recuperado de http://www2.inecc.gob. $\underline{\mathrm{mx} / \text { publicaciones/download/434.pdf }}$
Palomeque-De la Cruz, M. Á., Galindo-Alcántara, A., Escalona-Maurice, M. J., Ruiz-Acosta, S. D. C., Sánchez-Martínez, A. J., \& Pérez-Sánchez, E. (2017). Analysis of land use change in an urban ecosystem in the drainage area of the Grijalva river, México. Revista Chapingo. Serie Ciencias Forestales y del Ambiente, 23(1), 105-120. http://dx.doi.org/10.5154/r.rchscfa.2016.03.018

Pinkus-Rendón, M. J. y Contreras-Sánchez, A. (2012). Impacto socioambiental de la industria petrolera en Tabasco: el caso de la Chontalpa. LiminaR, 10(2), 122 144.

Recuperado de

http://www.scielo.org.mx/scielo.php?pid=S1665$80272012000200008 \&$ script=sci_arttext

Ramírez-García, P., J. López-Blanco, \& Ocana, D. (1998). Mangrove vegetation assessment in the Santiago River Mouth, Mexico, by means of supervised classification using LandsatTM imagery. Forest Ecology and Management, 105(1-3), 217-229. https://doi.org/10.1016/S0378-1127(97)00289-2

Ramos-Reyes, R., Palma-López, D. J., Ortiz-Solorio, C. A., Ortiz-García, C. F., y Díaz-Padilla, G. (2004). Cambios de uso de suelo mediante técnicas de sistemas de información geográfica en una región cacaotera. Terra Latinoamericana, 22(3), 267-278. Recuperado de http://www.redalyc.org/pdf/573/57322303.pdf

Ramos-Reyes, R., Sánchez-Hernández, R., y GamaCampillo, L. M. (2016). Análisis de cambios de uso del suelo en el municipio costero de Comalcalco, Tabasco, México. Ecosistemas y recursos agropecuarios, 3(8), 151-160. Recuperado de http://www.scielo.org.mx/scielo.php?pid=S2007$\underline{90282016000200151 \& \text { script=sci_arttext\&tlng=en }}$

Rawat, J. S. \& Kumar, M. (2015). Monitoring land use/cover change using remote sensing and GIS techniques: A case study of Hawalbagh block, district Almora, Uttarakhand, India. The Egyptian Journal of Remote Sensing and Space Science, 18(1), 77-84. https://doi.org/10.1016/j.ejrs.2015.02.002 
Rojas, C., Sepúlveda-Zúñiga, E., Barbosa, O., Rojas, O., y Martínez, C. (2015). Patrones de urbanización en la biodiversidad de humedales urbanos en Concepción metropolitano. Revista de Geografía Norte Grande, 61, 181-204. Recuperado de http://revistanortegrande.cl/wpcontent/uploads/2016/08/art10.pdf

Rosete-Vergés, F. A., Pérez-Damián, J. L., VillalobosDelgado, M., Navarro-Salas, E. N., Salinas-Chávez, E., y Remond-Noa, R. (2014). El avance de la deforestación en México 1976-2007. Madera y bosques, 20(1), 21$35 . \quad$ Recuperado de http://www.scielo.org.mx/scielo.php?pid=S1405$04712014000100003 \&$ script=sci_arttext\&tlng=pt

Rzedowski, J. (1978). La vegetación de México. México, DF: Limusa.

Sánchez, P.H., Ulloa, D. G. A., y Ricardo, A. L. (1998). Conservación y uso sostenible de los manglares del Caribe Colombiano. Bogotá, Colombia: Ministerio del Medio Ambiente.

Sánchez-Munguía, A. (2005). Uso del suelo agropecuario y deforestación en Tabasco 1950-2000. Villahermosa, México: Universidad Juárez Autónoma de Tabasco.

Schmook, B. \& Vance, C. (2009). Agricultural policy, market barriers, and deforestation: the case of Mexico's southern Yucatán. World Development, $37(5)$, 1015-1025. https://doi.org/10.1016/j.worlddev.2008.09.006

Secretaría de Energía, Recursos Naturales y Protección Ambiental, SERNAPAN. (2013). Programa estatal de ordenamiento ecológico 2013-2018. Villahermosa, México: COPLADET Recuperado de http://www.setab.gob.mx/php/copladet/doctos/Regi onales/3PROGRAMAESTATALDEORDENAMIENTOECOLOGICO. pdf

Smith Guerra, P., y Romero Aravena, H. (2009). Efectos del crecimiento urbano del Área Metropolitana de Concepción sobre los humedales de RocuantAndalién, Los Batros y Lenga. Revista de Geografía Norte Grande, 43, 81-93. Recuperado de http://www.scielo.cl/scielo.php?pid=S071834022009 000200005\&script=sci_arttext\&tlng=pt
Sun, G. \& Lockaby, B. G. (2012). Water quantity and quality at the urban-rural interface. Urban-Rural Interfaces: Linking People and Nature, 29-48. http://dx.doi.org/10.2136/2012.urban-rural.c3

Torbick, N. M., Qi, J., Roloff, G. J., \& Stevenson, R. J. (2006). Investigating impacts of land-use land cover change on wetlands in the Muskegon River Watershed, Michigan, USA. Wetlands, 26(4), 1103$1113 . \quad$ https://doi.org/10.1672/02775212(2006)26[1103:IIOLLC]2.0.CO;2

Torres-Vera, M. A., Prol-Ledesma, R. M., \& García-López, D. (2009). Three decades of land use variations in Mexico City. International Journal of Remote Sensing, 30(1),

117-138. http://dx.doi.org/10.1080/01431160802261163

West, R.C., Psuty, P., y Thom, B. (1976). Las Tierras Bajas de Tabasco en el Sureste de México. Villahermosa: Gobierno del Estado de Tabasco.

Yáñez-Arancibia, A., Day, J. W., Twilley, R. R., y Day, R. H. (2014). Manglares: ecosistema centinela frente al cambio climático, Golfo de México. Madera y bosques, 20(SPE), 39-75. Recuperado de http://www.scielo.org.mx/scielo.php?pid=S140504712014000500003\&script=sci_arttext

Zarazúa-Escobar, J. A., Almaguer-Vargas, G., y OcampoLedesma, J. G. (2011). El programa de apoyos directos al campo (PROCAMPO) y su impacto sobre la gestión del conocimiento productivo y comercial de la agricultura del Estado de México. Agricultura, sociedad y desarrollo, 8(1), 89-105. Recuperado de http://www.scielo.org.mx/scielo.php?script=sci_artte xt\&pid=S1870-54722011000100005

Zavala, J. y Castillo, A. O. (2007). Cambio de uso de la tierra en el estado de Tabasco. En D. J. Palma y A. Triano (Eds.), Plan de uso sustentable de los suelos del estado de Tabasco (vol, II, pp. 38-56). Villahermosa.

Zhang, L. \& Shao, H. (2013). Direct plant-plant facilitation in coastal wetlands: a review. Estuarine, Coastal and Shelf Science, 119, 1-6. https://doi.org/10.1016/j.ecss.2013.01.002 
Zepeda Gómez, C., Nemiga, X. A., Lot Helgueras, A., y Madrigal Uribe, D. (2012). Análisis del cambio del uso del suelo en las ciénegas de Lerma (1973-2008) y su impacto en la vegetación acuática. Investigaciones geográficas, 78, 48-61.

http://dx.doi.org/10.14350/rig.32469 